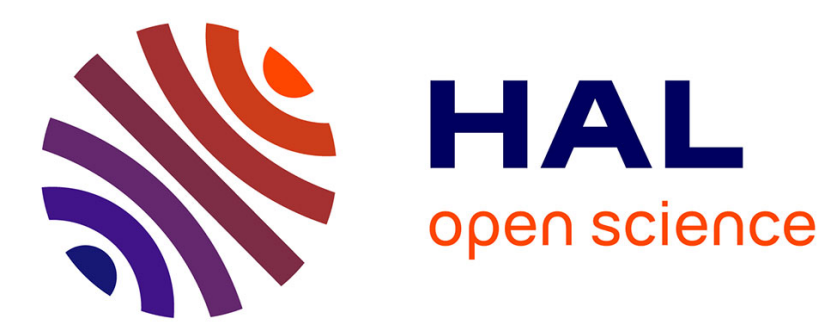

\title{
Real-time Teleoperation of Flexible Beveled-tip Needle Insertion using Haptic Force Feedback and 3D Ultrasound Guidance
}

\author{
Jason Chevrie, Alexandre Krupa, Marie Babel
}

\section{To cite this version:}

Jason Chevrie, Alexandre Krupa, Marie Babel. Real-time Teleoperation of Flexible Beveled-tip Needle Insertion using Haptic Force Feedback and 3D Ultrasound Guidance. ICRA 2019 - IEEE International Conference on Robotics and Automation, May 2019, Montreal, Canada. pp.1-7. hal-02053101

\author{
HAL Id: hal-02053101 \\ https://hal.inria.fr/hal-02053101
}

Submitted on 1 Mar 2019

HAL is a multi-disciplinary open access archive for the deposit and dissemination of scientific research documents, whether they are published or not. The documents may come from teaching and research institutions in France or abroad, or from public or private research centers.
L'archive ouverte pluridisciplinaire HAL, est destinée au dépôt et à la diffusion de documents scientifiques de niveau recherche, publiés ou non, émanant des établissements d'enseignement et de recherche français ou étrangers, des laboratoires publics ou privés. 


\title{
Real-time Teleoperation of Flexible Beveled-tip Needle Insertion using Haptic Force Feedback and 3D Ultrasound Guidance
}

\author{
Jason Chevrie ${ }^{1}$, Alexandre Krupa ${ }^{2}$, Marie Babel ${ }^{3}$
}

\begin{abstract}
Needle insertion procedures can greatly benefit from robotic systems to improve their accuracy and success rate. However, a fully automated system is usually not desirable and the clinicians need to be included in the control loop. In this paper we present a teleoperation framework for beveledtip flexible needle steering that enables the user to directly and intuitively control the trajectory of the needle tip via a haptic interface. The 6 degrees of freedom of the needle base are used to perform several automatic safety and targeting tasks in addition to the one controlled by the user. Real-time visual feedback is provided by a 3D ultrasound probe and used to track the 3D location of the needle and of a spherical target. Several haptic force feedback are compared as well as two different levels of mix between automated and user-controlled tasks. A validation of the framework is conducted in gelatin phantom and a mean targeting accuracy of $2.5 \mathrm{~mm}$ is achieved. The results show that providing an adequate haptic guidance to the user can reduce the risks of damage to the tissues while still letting the surgeon in control of the tip trajectory.
\end{abstract}

\section{INTRODUCTION}

Needle insertion is a widely performed medical procedure used for a large variety of applications, such as the treatment and diagnosis of cancers. Such procedures require a high accuracy in the placement of the needle tip to ensure the success of the operation and to avoid medical complications or misdiagnosis [1]. Many research work have focused on the modeling of the needle/tissue interaction to predict the deformation of the needle during the insertion [2], [3], [4]. Such models have then been used in robotic control frameworks to assist the surgeon and to improve the targeting accuracy [5], [6], [7]. Robots can indeed be of great help to perform repetitive gestures with consistency and accuracy.

Imaging modalities such as magnetic resonance imaging (MRI), computed tomography (CT) or ultrasound (US) are usually used to perform needle insertion procedures under visual guidance. In the case of MRI or CT, surgeons have a restricted access to the patient because of the bulky scanners which limit the size of the workspace or because of the ionizing radiations emitted during the $\mathrm{CT}$ image acquisition. On the contrary, with US the workspace is less limited and the insertion can be performed at the same time as the imaging process. However the accuracy of the needle insertion can be reduced by freehand probe holding since the surgeons have to coordinate the position of both the US probe and the needle [8]. In each case, teleoperated robotic systems can offer better operating conditions for the

J. Chevrie is affiliated with Univ Rennes, Inria, CNRS and IRISA, France. jason.chevrie@gmail.com.

M. Babel is affiliated with Univ Rennes, INSA, CNRS, Inria and IRISA, France. marie.babeleirisa.fr.

A. Krupa is affiliated with Univ Rennes, Inria, CNRS and IRISA, France. alexandre.krupa@inria.fr. surgeons, who do not need to be directly near the patient anymore to operate, but can be positioned in an ergonomic way with an optimized access to the different available intraoperative feedback modalities.

\section{A. Related work}

Many teleoperated robotic systems have been designed for needle insertion procedures [9], [10]. However, the surgeon is still often required to directly control the different degrees of freedom (DOF) of the robot, which can have various and complex effects on the actual tip trajectory. In addition, teleoperated robots that are designed to work in the limited workspace of MRI or CT are often controlled in a discontinuous manner due to the non real-time nature of the imaging process. The insertion is then performed step by step and alternates between image acquisition, correction of the needle base placement and insertion steps [11].

In the case of a continuous insertion whose velocity control is left to the surgeon, the robot motion is often limited to the insertion and rotation around the needle axis [12], [13], [10]. Continuous needle insertion usually requires the use of US imaging to obtain a fast visual feedback and automatic needle insertion under 2D or 3D US guidance have been the focus of many work [5], [14], [15], [16]. However there have been only few work on continuous needle steering under US guidance using all available DOF of the needle base [17], [18] and, to the best of our knowledge, none of them included the surgeon in the control loop for an robot teleoperation.

\section{B. Contributions}

In this paper we propose a preliminary study of a control framework that enables the real-time semi-automatic teleoperation of the needle tip 3D trajectory during its insertion in soft tissues. The framework uses all $6 \mathrm{DOF}$ of the needle base and intuitively gives the surgeon the control of the tip trajectory, while automatically performing an automatic orientation of the bevel and computing the optimal needle base velocity that eventually needs to be applied by the robot to achieve the desired tip motion. The insertion is performed using 3D US feedback in order to get a real-time tracking of both the needle and the target, which are considered as inputs for the controller, and to provide visual information to the surgeon. A haptic interface is used to get the input from the surgeon and to give an informative haptic force feedback. Different methods of computing the force feedback are here compared in terms of targeting performance and safety of the procedure.

This paper is divided as follows: the different constitutive parts of the control framework are presented in section II. 


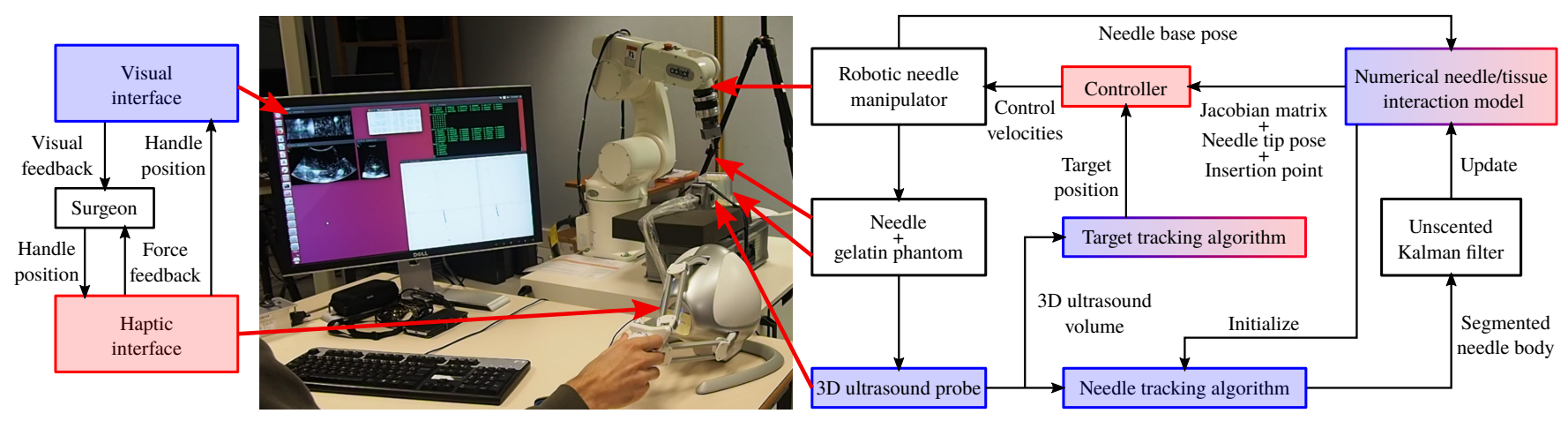

Fig. 1. Control framework and setup used to perform a teleoperated needle insertion. Elements in blue interact together to provide the visual interface: the target and needle tracking algorithms, the ultrasound probe and the needle/tissue interaction model send data to be displayed on the screen. Elements in red interact together to provide the haptic interface: the target tracking algorithm and the needle/tissue interaction model are used to compute the haptic feedback sent to the haptic device, while the haptic device sends position data to the controller.

The different strategies for the control and for the computation of the haptic feedback are also detailed. In section III we describe the experiments performed to validate the teleoperation framework and we discuss the results obtained from a comparative study of the different kinds of haptic feedback. Finally section IV provides conclusions on this work and directions for future work and improvement.

\section{Semi Autonomous InSERTION METHOdS}

This section describes the different elements of our semiautonomous teleoperation framework summarized in Fig. 1.

\section{A. Needle insertion framework}

We consider the case of a needle attached by its base to a robot arm. The control of the robot is performed using the task function framework [19]. Let $\boldsymbol{v}=[\mathbf{v} \boldsymbol{\omega}] \in \operatorname{se}(3)$ be the velocity screw vector applied to the needle base by the robot, with $\mathbf{v} \in \mathbb{R}^{3}$ the translation velocity and $\boldsymbol{\omega} \in \mathbb{R}^{3}$ the angular velocity. We compute $\boldsymbol{v}$ such that $N$ objective tasks can be fulfilled at the same time. Each task is described by a vector function $\boldsymbol{e}_{i} \in \mathbb{R}^{n_{i}}$ of dimension $n_{i}$. The task variation $\dot{\boldsymbol{e}}_{i}$ is linked to $\boldsymbol{v}$ by a time-varying Jacobian matrix $\boldsymbol{J}_{i}$ such that

$$
\dot{\boldsymbol{e}}_{i}=\boldsymbol{J}_{i} \boldsymbol{v} .
$$

In order to set the values of the tasks' variations to their desired values $\dot{e}_{i}^{d}$, the optimal velocity can be computed as

$$
\begin{gathered}
\boldsymbol{v}=\boldsymbol{J}^{+} \dot{\boldsymbol{e}}^{d}, \\
\text { with } \boldsymbol{J}=\left[\begin{array}{c}
\boldsymbol{J}_{1} \\
\vdots \\
\boldsymbol{J}_{N}
\end{array}\right] \quad \text { and } \quad \dot{\boldsymbol{e}}^{d}=\left[\begin{array}{c}
\dot{\boldsymbol{e}}_{1}^{d} \\
\vdots \\
\dot{\boldsymbol{e}}_{N}^{d}
\end{array}\right] \text {, }
\end{gathered}
$$

where ${ }^{+}$is a pseudo-inverse operator. In practice we use the damped pseudo-inverse [20] in order to avoid large velocities that can appear when $\boldsymbol{J}$ becomes near singular. The behavior of the controller then depends on the design of the different tasks. We choose here to design three tasks $(N=3)$ to control different aspects of the needle insertion.

The first task $e_{1}$ is used to control the safety aspect of the insertion. It is defined as the angle $\theta$, represented in Fig. 2, between the needle base axis and the insertion point $\boldsymbol{p}_{i n}$ at the surface of the tissue, such that

$$
\theta=-\operatorname{atan} 2\left(\sqrt{x_{i n}^{2}+y_{i n}^{2}}, z_{i n}\right),
$$

where $x_{i n}, y_{i n}$ and $z_{i n}$ are the coordinates of the insertion point $\boldsymbol{p}_{\text {in }}$ expressed in the needle base frame (see Fig. 2).

Using the geometric differenciation of $\theta$ with respect to $v$, the Jacobian matrix $\boldsymbol{J}_{\text {in }} \in \mathbb{R}^{1 \times 6}$ associated to the task $\boldsymbol{e}_{1}$ by (1) can be computed according to

$$
\boldsymbol{J}_{i n}=\left[\begin{array}{llllll}
-\frac{x_{i n} z_{i n}}{l d^{2}} & -\frac{y_{i n} z_{i n}}{l d^{2}} & \frac{l}{d^{2}} & \frac{y_{i n}}{l} & -\frac{x_{i n}}{l} & 0
\end{array}\right],
$$

where $d=\sqrt{x_{i n}^{2}+y_{i n}^{2}+z_{i n}^{2}}$ is the distance between the needle base and the insertion point and $l=\sqrt{x_{i n}^{2}+y_{i n}^{2}}$ is the lateral distance between the needle base axis and the insertion point, as illustrated in Fig. 2.

This task ensures that the needle base automatically stays aligned with the insertion point, hence limiting the tissue deformation near the surface. The desired variation $\dot{e}_{1}^{d}$ of the task is set to regulate $\theta$ toward zero according to

$$
\dot{e}_{1}^{d}=-\lambda_{\theta} \theta,
$$

where $\lambda_{\theta}$ is a positive gain that tunes the decrease rate of $\theta$.

The second task $\boldsymbol{e}_{2} \in \mathbb{R}^{3}$ is used to control the trajectory of the needle tip and is defined as the position of the tip. The Jacobian matrix $\boldsymbol{J}_{\text {tip }} \in \mathbb{R}^{3 \times 6}$ associated to the task $\boldsymbol{e}_{2}$ is defined such that

$$
\dot{e}_{2}=\mathbf{v}_{t i p}=\boldsymbol{J}_{t i p} \boldsymbol{v},
$$

where $\mathbf{v}_{t i p} \in \mathbb{R}^{3}$ is the velocity of the needle tip expressed in the tip frame. Note that $\boldsymbol{J}_{t i p}$ depends on the properties of the needle and of the tissues, such that a specific method needs to be used for its computation, as will be detailed in section II-B. This task is used to include the surgeon in the control loop via the haptic interface. In this study we will compare two different ways to compute the desired velocity $\mathbf{v}_{\text {tip }}^{d} \in \mathbb{R}^{3}$, to see if it is better to let the user control the 3 DOF of the tip or only the insertion speed.

In the first case, full control over the tip trajectory is given to the user and $\mathbf{v}_{\text {tip }}^{d}$ is computed according to

$$
\dot{e}_{2}^{d}=\mathbf{v}_{t i p}^{d}=\alpha^{t i p} \boldsymbol{R}_{h}\left(\boldsymbol{p}_{h}-\boldsymbol{p}_{h, 0}\right),
$$

where $\alpha$ is a positive scaling factor used to convert a distance into a velocity, ${ }^{t i p} \boldsymbol{R}_{h} \in S O(3)$ is the rotation matrix from the tip frame to the frame of the haptic device (see Fig. 3), $\boldsymbol{p}_{h}$ is the current position of the handle of the haptic device and 
$\boldsymbol{p}_{h, 0}$ is a default position at the center of the haptic device workspace, which will be used as a reference position for both the control law and the haptic feedback (see section IID.2). Using this kind of control law, the more the user moves the handle of the haptic device away from the default position, the higher is the velocity applied to the tip. The user can thus freely control the trajectory of the tip and they are in charge of ensuring that it is effectively driven toward the target.

In the second case, only the insertion velocity is controlled by the user, while the direction of the tip velocity is maintained oriented toward the target. $\mathbf{v}_{t i p}^{d}$ is then computed as

$$
\begin{aligned}
\dot{e}_{2}^{d} & =\mathbf{v}_{\text {tip }}^{d}=V_{t} \frac{\boldsymbol{p}_{\text {tar }}}{\left\|\boldsymbol{p}_{\text {tar }}\right\|}, \\
\text { with } \quad V_{t} & =\alpha\left({ }^{t i p} \boldsymbol{R}_{h}\left(\boldsymbol{p}_{h}-\boldsymbol{p}_{h, 0}\right)\right) . \boldsymbol{d}_{t i p},
\end{aligned}
$$

where $V_{t}$ is the amplitude of the tip velocity, $\boldsymbol{p}_{\text {tar }} \in \mathbb{R}^{3}$ is the position of the target and $\boldsymbol{d}_{t i p} \in \mathbb{R}^{3}$ is the direction of the tip axis (i.e. $z_{t}$ in Fig. 2), both expressed in the tip frame. The user can thus choose the insertion velocity $V_{t}$, while the system automatically adapts the tip trajectory to reach the target. Note that the dot product with $\boldsymbol{d}_{t i p}$ in (10) ensures that only the component of the motion of the handle along the tip direction is taken into account to compute the norm of the applied tip velocity, while lateral components are discarded in order to keep visual consistency. This way $V_{t}$ can also be negative if the user wants to retract the needle.

Finally the third task $e_{3}$ is used to automatically ensure that the circular tip trajectory induced by the bevel during the insertion is oriented in the desired direction, reducing the needle bending required to obtain the tip motion. It is defined as the angle $\gamma$, represented in Fig. 2, between the desired tip velocity $\mathbf{v}_{\text {tip }}^{d}$ and the cutting-edge of the bevel:

$$
e_{3}=\gamma=\operatorname{atan} 2\left(v_{t i p, y}^{d}, v_{t i p, x}^{d}\right)-\frac{\pi}{2},
$$

where $v_{t i p, x}^{d}$ and $v_{t i p, y}^{d}$ are the components of $\mathbf{v}_{t i p}^{d}$ along the $\boldsymbol{x}_{t}$ and $\boldsymbol{y}_{t}$ axis of the tip frame, respectively (see Fig. 2). Assuming a torsionally stiff needle, the rotation of the needle base around the needle shaft is directly transmitted to the needle tip, such that the Jacobian matrix $\boldsymbol{J}_{b} \in \mathbb{R}^{1 \times 6}$ associated to the task $\boldsymbol{e}_{3}$ by (1) can be computed as

$$
\boldsymbol{J}_{b}=\left[\begin{array}{llllll}
0 & 0 & 0 & 0 & 0 & 1
\end{array}\right] \text {. }
$$

The desired variation $e_{3}^{d}$ of the task is set such that $\gamma$ is always regulated toward zero according to

$$
\dot{e}_{3}^{d}=-\lambda_{\gamma} \gamma,
$$

where $\lambda_{\gamma}$ is a positive gain that tunes the decrease rate of $\gamma$.

The final velocity screw vector applied to the needle base is then computed using (2). The control law enables the user to control the state of the insertion in real-time, while leaving the complexity of the needle base manipulation to the system.

The Jacobian matrices associated to the first and third tasks can be computed based on geometry. However, the Jacobian matrix $\boldsymbol{J}_{\text {tip }}$ associated to the second task needs to be estimated using a model of the needle interaction with the tissues. We describe in the following section II-B the mechanics-based model that we use to compute $\boldsymbol{J}_{t i p}$.

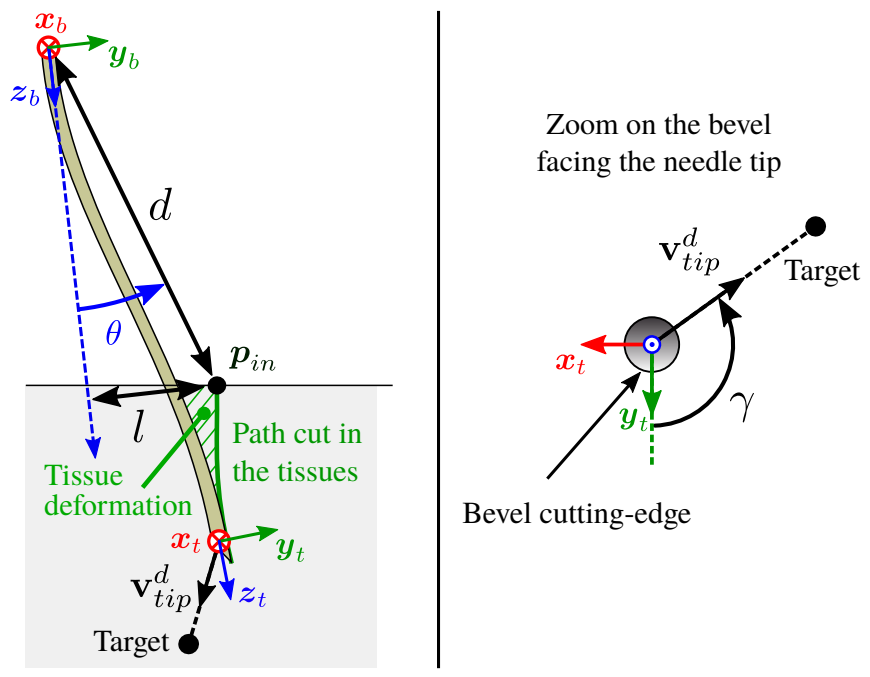

Fig. 2. Illustration of the different elements used in the task functions for the control: angle $\theta$ between the needle base axis and the initial insertion point $\boldsymbol{p}_{\text {in }}$, angle $\gamma$ between the cutting edge of the bevel and desired tip velocity $\mathbf{v}_{\text {tip }}^{d}$. Tissue deformation is computed as the mean displacement of the tissue from its initial position along the inserted part of the needle.

\section{B. Needle insertion modeling}

The 6 DOF of the needle base are controlled in this framework, so that lateral motions can be applied to the base. Due to the needle flexibility and its interaction with the tissues all along its shaft, the needle shape can be modified inside and outside of the tissues, so that the widely used kinematic models of unicycle [21] can not be used here.

Instead, we model the whole needle body in order to estimate the effect of the needle base motion on the tip motion. We use the same model as was introduced in [4], consisting of a flexible beam representing the needle that interacts with a second beam representing the path cut in the tissues by the beveled tip (see Fig 2). This second beam is updated online to take into account the non-linear properties and the external motions of the tissues using an unscented Kalman filter. In [4] CCD cameras were used to observe the needle through translucent gelatin and it was shown that good estimation performances with mean accuracy under $1 \mathrm{~mm}$ could be obtained even when the acquisition rate was lowered to $1 \mathrm{~Hz}$. Here we use instead 3D US imaging to observe the needle in non translucent soft tissues, as this medical imaging modality is commonly used in medical practice to perform needle insertion procedures. The position of several points along the needle shaft, necessary for the model update, are computed using the needle tracking algorithm in 3D US volumes presented in the following section II-C.

A finite difference method is then used with the model to compute the Jacobian matrix $J_{t i p}$ linking the motion of the needle base to the tip velocity as defined in (7).

\section{Needle and target tracking in 3D ultrasound}

As the needle and target tracking is not the main focus of this paper, we only briefly describe the principle of the algorithms that we use. More details can be found in [22]. The needle is tracked in each US volume using an iterative algorithm to locally fit a polynomial curve to the best location of the needle. The curve is first initialized using the current 
state of the model presented in section II-B. At each iteration, the curve is deformed to maximize an objective function that is computed from the voxel intensity around the curve and that takes into account the typical needle-induced artifacts in the US images. A spherical target is also tracked in the 3D US volumes using a 3D version of a Star algorithm [23].

\section{Teleoperation and user interface}

In order to close the control loop and to ensure the success of the needle insertion, feedback needs to be provided to the user all along the procedure. Visual and haptic feedback are usually used together to provide complementary information on the state of the insertion. In the following we describe the visual and haptic information that we provide to the user in order to perform the teleoperated needle insertion.

1) Visual feedback: In order to monitor the good proceeding of the insertion, a visual feedback is provided to the user in the form of three orthogonal slices of the last acquired US volume, as can be seen in Fig. 3. The slices are automatically selected such that they intersect each other at the current location of the tip of the needle model, such that the tip is always visible during the insertion. Additional information are also displayed on each view in order to help the surgeon interpreting the images and performing the insertion toward the target. The needle model is projected onto each view to give the currently estimated position of the needle body. The results of the needle and target tracking algorithms are also projected on each view, so that it is easy to identify the direction in which the needle should go to reach the target.

Since the controller defined in section II-A applies a tip velocity that is proportional to the position of the handle of the haptic device, it is important to provide a visual feedback of this position. The point corresponding to the default position $\boldsymbol{p}_{h, 0}$ of the haptic device is mapped on each view such that it corresponds to the current position of the needle tip. The position $\boldsymbol{p}_{h}$ of the handle is then displayed on each image using a similar method as was used in [24], i.e. using a cross with a size equal to the orthogonal distance of $\boldsymbol{p}_{h}$ from the plane and with a color that depends on which side of the image plane $\boldsymbol{p}_{h}$ is lying. A transformation is first applied to $\boldsymbol{p}_{h}$ such that the motion applied by the user on the haptic device is aligned with the motion of the cross in the XY view of the US volume (see Fig. 3). This leads to an intuitive control of the direction of the tip velocity in the XY view, independently of the real orientation of the US probe, haptic device or needle in the world frame. Note that the cross will move at the same speed as the needle tip if the user does not change the position of the haptic device.

2) Haptic feedback: We compare three different kinds of force feedback to provide various levels of guidance to the user during the insertion. Each feedback consists in a force $\boldsymbol{f}_{h} \in \mathbb{R}^{3}$ that drives the handle of the haptic device toward its default position $\boldsymbol{p}_{h, 0}$ defined previously in (8). The applied force is linked to the current position $\boldsymbol{p}_{h}$ of the haptic device by a positive definite stiffness matrix $\boldsymbol{K}_{h} \in \mathbb{R}^{3 \times 3}$ such that

$$
\boldsymbol{f}_{h}=-\boldsymbol{K}_{h}\left(\boldsymbol{p}_{h}-\boldsymbol{p}_{h, 0}\right) \text {. }
$$

Using this method, the intensity of the feedback provided to the user is thus proportional to the velocity that is currently

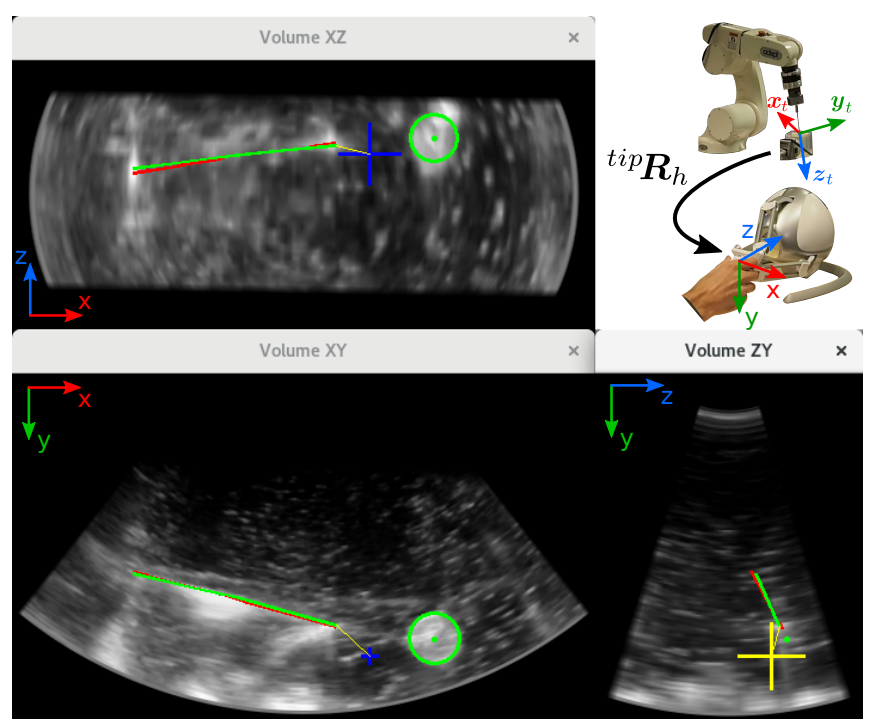

Fig. 3. Picture of the screen view providing the visual feedback from the ultrasound probe. Three orthogonal slices of the current US volume passing by the model needle tip are displayed. The needle model is represented by the green lines, the needle tracking is represented by the red lines and the target center and boundaries are represented by the green dots and green circles. The current position of the haptic device is represented by blue crosses when it is in front of the image plane and by yellow crosses when it is behind the image plane, and the size of the crosses corresponds to the orthogonal distance from the image plane.

applied to the needle tip by the controller defined in (8). This ensures the safety of the framework by natural driving the haptic device toward a position where no tip velocity is applied by the controller. Depending on the exact definition of $\boldsymbol{K}_{h}$, which can be non-diagonal, different guiding effects can be created by applying a different stiffness in different directions. We describe in the following the three different methods used to define $\boldsymbol{K}_{h}$ and represented in Fig. 4.

a) Method 1: We define a stiffness matrix $\boldsymbol{K}_{h}^{i s o}$ such that it provides an isotropic stiffness, i.e. the force provided to the user is directed toward the haptic center and is proportional to the distance from this center. $\boldsymbol{K}_{h}^{i s o}$ is thus defined as a diagonal matrix with a constant factor $k$ in the diagonal. This way the feedback is directly proportional to the velocity applied to the tip. Using this definition, the user has a full control over the trajectory of the needle tip and does not feel constrained in any direction. However, no haptic information is provided on the target position or on the degree of feasibility of the tip motion.

b) Method 2: We define a stiffness matrix $\boldsymbol{K}_{h}^{t i p}$ such that the stiffness is anisotropic with a greater stiffness applied in directions in which it is difficult to move the tip without deforming the tissues and with a low stiffness in the other directions. This allows the user to be naturally guided in the direction in which the needle tip can be inserted without bending the needle and pushing laterally on the tissues. This method should increase the safety of the insertion procedure by limiting the lateral motions of the needle.

We use the Jacobian matrix $\boldsymbol{J}_{t i p, t}$ linking the base velocity to the tip velocity to identify the manipulability ellipsoid at the tip [25]. $\boldsymbol{J}_{t i p, t}$ is directly extracted from the left half of the Jacobian matrix $\boldsymbol{J}_{t i p}$ computed in (7). The manipulability ellipsoid can be characterized by three unitary orthogonal 
axis $\boldsymbol{u}_{i} \in \mathbb{R}^{3}$ each associated to one of the singular values $\sigma_{i}$ of $\boldsymbol{J}_{t i p, t}$. A low value for $\sigma_{i}$ indicates that moving the tip along axis $\boldsymbol{u}_{i}$ requires a large motion of the base and vice $v e r s a$. Due to the high axial stiffness of the needle, a direction for which the base velocity is transmitted to the tip always exists, so that one of the $\sigma_{i}$ should always be equal to 1 and the associated $\boldsymbol{u}_{i}$ indicates the natural insertion direction of the tip. Then we associate a stiffness $k_{i}$ for each axis such that

$$
k_{i}=\frac{k}{\epsilon+\sigma_{i}},
$$

where $k$ is the default isotropic stiffness defined previously and $\epsilon<1$ is used to bound the value of the stiffness when $\sigma_{i}$ becomes too small. This way, the natural insertion direction is associated to a low stiffness near $k$, while the other directions are associated to a stiffness that can grow up to a maximum value of $\frac{k}{\epsilon}$. We empirically chose $\epsilon=0.1$ in the experiments, which was enough to provide guidance without inducing stability issues in the haptic force feedback. The stiffness matrix $\boldsymbol{K}_{h}^{t i p}$ can then be computed as

$$
\boldsymbol{K}_{h}^{t i p}={ }^{t i p} \boldsymbol{R}_{h}^{T} \boldsymbol{U}\left[\begin{array}{ccc}
k_{1} & 0 & 0 \\
0 & k_{2} & 0 \\
0 & 0 & k_{3}
\end{array}\right] \boldsymbol{U}^{T}{ }^{t i p} \boldsymbol{R}_{h},
$$

where $\boldsymbol{U} \in \mathbb{R}^{3 \times 3}$ is a matrix containing the column vectors $\boldsymbol{u}_{i}$ and ${ }^{t i p} \boldsymbol{R}_{h}$ was defined in (8).

Using such a haptic feedback, the user can feel which direction is likely to cause the least bending of the needle and the least tissue deformation. At the beginning of the insertion lateral motions of the tip can be easily achieved, so that the applied stiffness is almost isotropic and the user is not much constrained. On the contrary, as the insertion depth increases, lateral motions of the tip require more lateral base motions, so that the user feels more guided in a specific direction which is the natural insertion direction of the needle.

c) Method 3: We define the anisotropic stiffness matrix $\boldsymbol{K}_{h}^{t a r}$ in a similar way to Method 2, except that the low stiffness axis is realigned with the target (see Fig. 4):

$$
\boldsymbol{K}_{h}^{t a r}={ }^{t a r} \boldsymbol{R}_{h}^{T} \boldsymbol{U}\left[\begin{array}{ccc}
k_{1} & 0 & 0 \\
0 & k_{2} & 0 \\
0 & 0 & k_{3}
\end{array}\right] \boldsymbol{U}^{T}{ }^{{ }^{t a r}} \boldsymbol{R}_{h},
$$

where ${ }^{t a r} \boldsymbol{R}_{h} \in S O(3)$ is the rotation matrix from the tip frame realigned with the target to the frame of the haptic device and the other terms were defined in (16). Using this feedback the user is naturally guided toward the target during the insertion.

\section{EXPERIMENTS}

In this section we describe the experiments that we performed to validate the teleoperation framework and we present the results of the comparison between the different control and feedback methods.

\section{A. Experimental setup}

The setup used for the experiments can be seen in Fig. 1. We used a Chiba biopsy needle (Angiotech MCN2208) with internal diameter $0.48 \mathrm{~mm}$ and external diameter $0.7 \mathrm{~mm}$ attached to the end effector of a 6 DOF manipulator
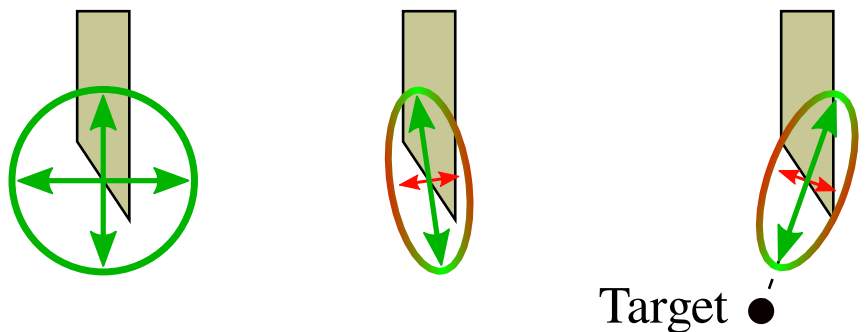

Fig. 4. Representation of the different definitions of the stiffness for the haptic force feedback, overlaid on the needle tip. From left to right: isotropic stiffness (Method 1), anisotropic stiffness in the tip direction (Method 2) and anisotropic stiffness in the target direction (Method 3). The ellipses represent a set of velocity vector that would induce a force feedback of constant intensity. Green and red arrows indicate velocity directions in which the intensity of the provided force feedback is low or high, respectively.

Viper S650 (Adept). The length of the needle that can bend is $12.6 \mathrm{~cm}$ long. The needle Young's modulus is assumed to be that of steel, i.e. $200 \mathrm{GPa}$. The insertion is done in a homemade porcine gelatin phantom embedding several spherical targets. Visual feedback is provided to the controller using a SonixTOUCH Research 3D US scanner (Ultrasonix Medical Corporation) with a 4DC7-3/40 motorized 3D US probe maintained fix during the experiments. The acquisition parameters are set such that a volume has a depth of $8 \mathrm{~cm}$, a frame field of view of $78^{\circ}$ and a wobbling angle of $37^{\circ}$. A new volume is acquired every $408 \mathrm{~ms}$. The US station allows us to get access to the prescan data acquired by the probe. The 3D volumes are reconstructed in cartesian coordinates [26] after a $3 \times 3 \times 3$ median filter is applied to remove some noise in the prescan data. A Falcon haptic interface (Novint Technologies) is used to get the user input and to provide the haptic feedback.

\section{B. Experimental scenario}

We compare four different combinations of user tasks and haptic feedback. The first three combinations use the 3 DOF user task (see (8)) along with one of the methods to compute the haptic feedback defined in section II-D.2 (with the same indexes). The fourth combination (Method 4) uses the user task limited to the insertion velocity (see (9)) along with the isotropic stiffness Note that the anisotropic stiffness is not considered in this method since the insertion direction is already imposed by the controller. In each case the default stiffness is set to $k=500 \mathrm{~N} \cdot \mathrm{m}^{-1}$ and the position to velocity scaling factor is set to $\alpha=0.1 s^{-1}$. For each insertion, the needle tip is first placed at the surface of the tissues and the interaction model is initialized. The needle is then inserted $8 \mathrm{~mm}$ into the tissues so that the user can initialize the needle and target tracking algorithms in the US volume displayed on the screen. The user can then start and stop the controller by clicking on a button on the haptic device.

\section{Results}

In this section we present and discuss the results of the experiments. Five insertions were performed for each method. Trajectories obtained during one of the insertions performed with Method 1 are shown in Fig. 5. We can see that the user is able to reach the target at the end of the insertion. Note that the trajectory obtained by integrating the 
$\mathrm{X}$

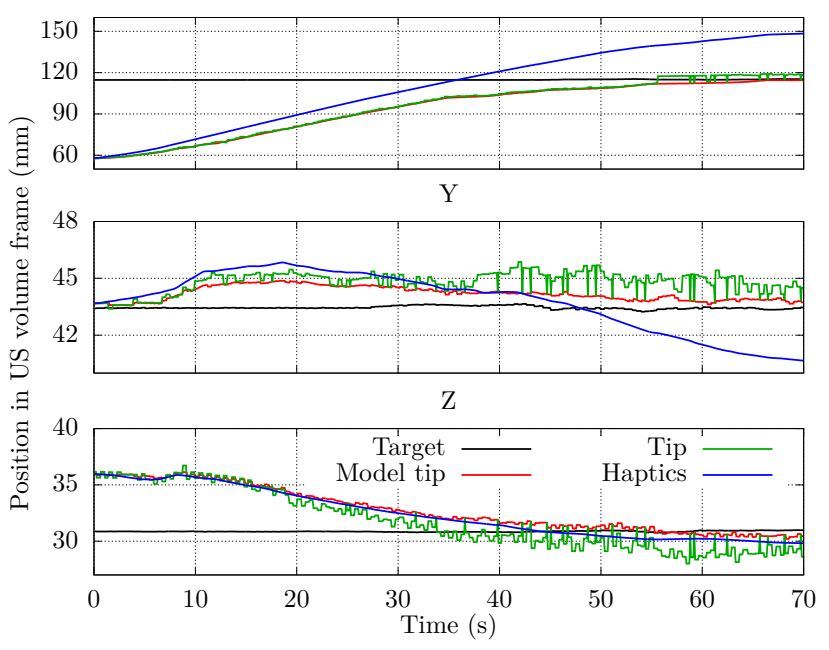

Fig. 5. Trajectories in the frame of the ultrasound probe during a typical example of insertion using the 3 DOF tip control and without haptic guidance (isotropic stiffness). The black curve represents the trajectory of the target, the red curve is the trajectory of the tip of the needle model, the green curve is the trajectory of the tracked needle tip and the blue curve is the position obtained by integrating the velocity input given by the user through the haptic interface.

TABLE I

COMPARISON OF THE FINAL TARGETING ERROR

\begin{tabular}{|c|c|c|c|} 
Method & Axial error $(\mathrm{mm})$ & \multicolumn{1}{c|}{ Lateral error $(\mathrm{mm})$} & Total error $(\mathrm{mm})$ \\
\hline 1 & $1.3 \pm 0.7$ & $1.7 \pm 0.5$ & $2.5 \pm 0.6$ \\
\hline 2 & $-0.4 \pm 1.5$ & $1.5 \pm 0.5$ & $2.0 \pm 0.6$ \\
\hline 3 & $0.1 \pm 1.8$ & $1.6 \pm 0.4$ & $2.3 \pm 0.5$ \\
\hline 4 & $-1.3 \pm 1.1$ & $2.0 \pm 0.6$ & $2.5 \pm 1.0$ \\
\hline
\end{tabular}

input of the user diverges from the actual trajectory of the tip, mainly due to the damping introduced in the control law (2). However this does not seem to hinder the targeting performance since the visual loop is closed by the user.

We first compare the performance in terms of targeting accuracy, segmented manually in the US volume at the end of the insertion. Table I gives the mean final targeting accuracy obtained across the 5 insertions for each method. Good targeting performance can be obtained with all methods, with a mean targetting error under $2.5 \mathrm{~mm}$ and a mean lateral error under $2.0 \mathrm{~mm}$, which is on par with the accuracy obtained in current research work on needle steering under US feedback and is sufficient for most medical applications.

In order to increase the safety of the insertion procedure, it is important to avoid breaking the needle or tearing the tissues. We thus compared the mean and maximum values of the needle bending and of the tissue deformation obtained during the insertion with the different methods. The needle bending is taken as the integral of the absolute value of the needle curvature along the whole needle shaft. The tissue deformation is taken as the mean displacement of the tissue from its initial position along the inserted needle (see Fig. 2). Both are estimated using the current state of the interaction model presented in section II-B. The average and maximum values obtained for each method are summarized in Table II.

As expected, the amount of needle bending and tissue deformation is greatly reduced when the tip motion is fully constrained by the controller (Method 4) compared to the
TABLE II

COMPARISON OF THE NEEDLE BENDING AND TISSUE DEFORMATION

\begin{tabular}{|c|c|c|c|c|}
\hline \multirow{2}{*}{ Method } & \multicolumn{2}{|c|}{ Bending $\left(m^{-1}\right)$} & \multicolumn{2}{|c|}{ Tissue deformation $(\mathrm{mm})$} \\
\hline & Mean & Max & Mean & Max \\
\hline 1 & 0,038 & 0,301 & 0.58 & 4.0 \\
\hline 2 & 0,045 & 0,575 & 0.63 & 4.2 \\
\hline 3 & 0,022 & 0,339 & 0.45 & 3.6 \\
\hline 4 & 0,004 & 0,0698 & 0.38 & 2.0 \\
\hline
\end{tabular}

3 DOF control. This is mainly due to the fact that the controller aligns the needle with the target as soon as the insertion begins, so that little lateral motion of the needle base is then required for the remaining of the insertion. Guiding the user toward the target (Method 3) also seems to decrease the induced amount of needle bending and tissue deformation compared to the non-guided Method 1. This validates the usefulness of providing a haptic force feedback to the user to guide the insertion. However, contrary to what could be expected, guiding the user along the direction of least lateral needle base motion (Method 2) seems to actually increase the amount of induced needle bending and tissue deformation. This can actually be explained by the same reasons that justified the good behavior of Method 4. Using this kind of guidance at the beginning of the insertion will indeed tend to delay the alignment of the needle with the target, which will then require more tissue deformation to be achieved later when the needle is deeper inserted.

Overall the results validate the good performance of the framework in terms of targeting accuracy and stress out the importance of carefully choosing the way haptic guidance is provided to the surgeon in order to ensure a safe procedure.

\section{CONCLUSIONS AND FUtURE WORK}

In this paper we presented a proof-of-concept for a teleoperation framework to perform the real-time robotic insertion of a beveled-tip flexible needle under 3D ultrasound guidance and haptic feedback. The framework enables the user to intuitively guide the trajectory of the needle tip in the image while the controller handles the complexity of the $6 \mathrm{D}$ motion that needs to be applied to the needle base. A mean targeting accuracy of $2.5 \mathrm{~mm}$ could be achieved in gelatin phantoms, validating the whole framework. We compared different ways to provide the haptic feedback as well as different levels of control given to the user on the tip trajectory. Limiting the user input to the insertion speed while automatically controlling the trajectory of the needle tip seems to provide a safer insertion process, however it may be too constraining and can not handle situations where more control over the tip trajectory is required, for example if unpredicted obstacles need to be avoided. On the contrary, giving the full control of the 3D tip velocity to the user and applying a haptic feedback to guide the user toward the target proved to maintain a low level of needle bending and tissue deformation.

Since the results are user-dependent, future work would include a larger scale user study with experts and beginners in order to confirm the obtained performance. As the different components of the framework can already handle the effect of external tissue motion with little to no adaptation, tests should also be conducted to validate its ability to perform automatic motion compensation during the teleoperation. 


\section{REFERENCES}

[1] N. Abolhassani, R. Patel, and M. Moallem, "Needle insertion into soft tissue: A survey," Medical Engineering \& Physics, vol. 29, no. 4, pp. 413 - 431, 2007. [Online]. Available: http://www.sciencedirect.com/science/article/pii/S1350453306001457

[2] S. Misra, K. Reed, B. Schafer, K. Ramesh, and A. Okamura, "Mechanics of flexible needles robotically steered through soft tissue," The International Journal of Robotics Research, 2010. [Online]. Available: http://ijr.sagepub.com/content/early/2010/06/02/0278364910369714.abstract

[3] M. Khadem, C. Rossa, N. Usmani, R. Sloboda, and M. Tavakoli, "A two-body rigid/flexible model of needle steering dynamics in soft tissue," IEEE/ASME Transactions on Mechatronics, vol. PP, no. 99, pp. 1-1, 2016.

[4] J. Chevrie, A. Krupa, and M. Babel, "Online prediction of needle shape deformation in moving soft tissues from visual feedback," in 2016 IEEE/RSJ International Conference on Intelligent Robots and Systems (IROS), Oct 2016, pp. 2375-2380.

[5] M. Abayazid, R. Roesthuis, R. Reilink, and S. Misra, "Integrating deflection models and image feedback for real-time flexible needle steering," Robotics, IEEE Transactions on, vol. 29, no. 2, pp. 542553, April 2013.

[6] M. Waine, C. Rossa, R. Sloboda, N. Usmani, and M. Tavakoli, "Needle tracking and deflection prediction for robot-assisted needle insertion using 2d ultrasound images," Journal of Medical Robotics Research, vol. 01, no. 01, p. 1640001, 2016. [Online]. Available: http://www.worldscientific.com/doi/abs/10.1142/S2424905X16400018

[7] Y. Adagolodjo, L. Goffin, M. de Mathelin, and H. Courtecuisse, "Inverse real-time finite element simulation for robotic control of flexible needle insertion in deformable tissues," in 2016 IEEE/RSJ International Conference on Intelligent Robots and Systems (IROS), Oct 2016, pp. 2717-2722.

[8] D. Kaye, D. Stoianovici, and M. Han, "Robotic ultrasound and needle guidance for prostate cancer management: review of the contemporary literature," Current opinion in urology, vol. 24, no. 1, pp. 75-80, 2014.

[9] O. Piccin, L. Barb, B. Bayle, M. de Mathelin, and A. Gangi, "A force feedback teleoperated needle insertion device for percutaneous procedures," The International Journal of Robotics Research, vol. 28, no. 9, pp. 1154-1168, 2009. [Online]. Available: https://doi.org/10.1177/0278364909101408

[10] P. Moreira, G. van de Steeg, T. Krabben, J. Zandman, E. Hekman, F. van der Heijden, R. Borra, and S. Misra, "The miriam robot: A novel robotic system for mr-guided needle insertion in the prostate," Journal of Medical Robotics Research, vol. 2, no. 3, p. 1750006, 2017. [Online]. Available: http://www.worldscientific.com/doi/abs/10.1142/S2424905X17500064

[11] A. A. Goldenberg, J. Trachtenberg, W. Kucharczyk, Y. Yi, M. Haider, L. Ma, R. Weersink, and C. Raoufi, "Robotic system for closedbore mri-guided prostatic interventions," IEEE/ASME Transactions on Mechatronics, vol. 13, no. 3, pp. 374-379, June 2008

[12] C. Pacchierotti, M. Abayazid, S. Misra, and D. Prattichizzo, "Teleoperation of steerable flexible needles by combining kinesthetic and vibratory feedback," IEEE Transactions on Haptics, vol. 7, no. 4, pp. 551-556, Oct.-Dec. 2014. [Online]. Available: doi.ieeecomputersociety.org/10.1109/TOH.2014.2360185

[13] R. Seifabadi, F. Aalamifar, I. Iordachita, and G. Fichtinger, "Toward teleoperated needle steering under continuous mri guidance for prostate percutaneous interventions," The International Journal of Medical Robotics and Computer Assisted Surgery, vol. 12, no. 3, pp. 355-369, 2016. [Online]. Available: http://dx.doi.org/10.1002/rcs.1692

[14] T. Adebar, A. Fletcher, and A. Okamura, "3d ultrasound-guided robotic needle steering in biological tissue," Biomedical Engineering, IEEE Transactions on, vol. PP, no. 99, pp. 1-1, 2014.

[15] P. Chatelain, A. Krupa, and N. Navab, "3d ultrasound-guided robotic steering of a flexible needle via visual servoing," in Robotics and Automation (ICRA), 2015 IEEE International Conference on, Seattle, WA, May 2015, pp. 2250-2255.

[16] P. Mignon, P. Poignet, and J. Troccaz, "Beveled-tip needle-steering using 3d ultrasound, mechanical-based kalman filter and curvilinear roi prediction," in 2016 14th International Conference on Control, Automation, Robotics and Vision (ICARCV), Nov 2016, pp. 1-6.

[17] Z. Neubach and M. Shoham, "Ultrasound-guided robot for flexible needle steering," Biomedical Engineering, IEEE Transactions on, vol. 57, no. 4, pp. 799-805, April 2010.

[18] J. Chevrie, N. Shahriari, M. Babel, A. Krupa, and S. Misra, "Flexible needle steering in moving biological tissue with motion compensation using ultrasound and force feedback," IEEE Robotics and Automation Letters, vol. 3, no. 3, pp. 2338-2345, July 2018.
[19] B. Siciliano and J.-J. Slotine, "A general framework for managing multiple tasks in highly redundant robotic systems," in Advanced Robotics, 1991. 'Robots in Unstructured Environments', 91 ICAR., Fifth International Conference on, June 1991, pp. 1211-1216 vol.2.

[20] A. Deo and I. Walker, "Overview of damped least-squares methods for inverse kinematics of robot manipulators," Journal of Intelligent and Robotic Systems, vol. 14, no. 1, pp. 43-68, 1995. [Online]. Available: http://dx.doi.org/10.1007/BF01254007

[21] R. Webster III, J. Kim, N. Cowan, G. Chirikjian, and A. Okamura, "Nonholonomic modeling of needle steering," The International Journal of Robotics Research, vol. 25, no. 5-6, pp. 509-525, 2006 [Online]. Available: http://ijr.sagepub.com/content/25/5-6/509.abstract

[22] J. Chevrie, "Flexible Needle Steering using Ultrasound Visual Servoing," Theses, Université de Rennes 1, Dec. 2017. [Online]. Available: https://tel.archives-ouvertes.fr/tel-01663761

[23] N. Friedland and D. Adam, "Automatic ventricular cavity boundary detection from sequential ultrasound images using simulated annealing," IEEE Transactions on Medical Imaging, vol. 8, no. 4, pp. 344-353, Dec 1989.

[24] W. Xia, S. West, M. Finlay, J.-M. Mari, S. Ourselin, A. David, and A. Desjardins, "Looking beyond the imaging plane: $3 \mathrm{~d}$ needle tracking with a linear array ultrasound probe," Tech. Rep. 1, 2017. [Online]. Available: https://doi.org/10.1038/s41598-017-03886-4

[25] T. Yoshikawa, "Manipulability of robotic mechanisms," The International Journal of Robotics Research, vol. 4, no. 2, pp. 3-9, 1985. [Online]. Available: https://doi.org/10.1177/027836498500400201

[26] D. Lee and A. Krupa, "Intensity-based visual servoing for non-rigid motion compensation of soft tissue structures due to physiological motion using 4d ultrasound," in Intelligent Robots and Systems (IROS), IEEE/RSJ International Conference on, Sept 2011, pp. 2831-2836. 